\title{
Eficiencia técnica de las entidades federativas de México
}

\section{Technical efficiency of the federal states in Mexico}

\author{
Osvaldo Becerril-Torres* \\ INMACULADA ÁLVAREZ-Ayuso** \\ Laura del Moral-Barrera*
}

\begin{abstract}
The interest in having a measurement of the technical efficiency arises from the acceptance of the existence of a breach in the theoretical asemptions of the total technical efficiency and in the empirical reality, so there is opportunity to improve the output only optimizing the inputs. In this context, the objective of this academic research is to obtain an indicator of levels of technical efficiency that allows learning the relative position and temporary evolution in the federal states of Mexico in respect to its efficient frontier. In particular, the frontier of production is approached by means of the analysis of stochastic frontiers stated by Battese and Coelli. The general result permits indentifying that production might be broadened in circa 20\%; likewise, a ranking of technical efficiency for the federal states of Mexico is made.
\end{abstract}

Keywords: stochastic production function; technical efficiency; analysis of stochastic frontiers; federal states.

\section{Resumen}

El interés por tener una medida de la eficiencia técnica parte de que se acepta la existencia de una brecha entre el supuesto teórico de eficiencia técnica total y la observada en la realidad empírica, por lo que se abre la posibilidad de mejorar el output únicamente optimizando los inputs. En este contexto, el objetivo de esta investigación es obtener un indicador de niveles de eficiencia técnica que permita identificar la posición relativa y la evolución temporal en las entidades federativas de México respecto a su frontera eficiente. En particular, se aproxima la frontera de producción mediante el análisis de fronteras estocásticas planteado por Battese y Coelli. El resultado general permite identificar que se puede ampliar la producción en aproximadamente $20 \%$. Asimismo, se elabora un ranking de eficiencia técnica para las entidades federativas de México.

Palabras clave: función de producción estocástica, eficiencia técnica, análisis de fronteras estocásticas, entidades federativas.

*Universidad Autónoma del Estado de México. Correos-e: obecerrilt@uaemex.mx, lauraelena_toluca1@yahoo.com.mx.

**Universidad Autónoma de Madrid. Correo-e: inmaculada.alvarez@uam.es. 


\section{Introducción}

El concepto de eficiencia técnica es antiguo en economía, pero el interés por medirla no. Esto quizá se deba al hecho de que la teoría de producción neoclásica presupone eficiencia técnica total. En este sentido, muchas veces se asume que se conoce la función de producción y que la empresa es completamente eficiente. En la práctica, la función se desconoce y la evidencia empírica muestra que existen brechas entre la producción potencial y la real, es decir, el uso de los factores productivos es ineficiente.

Por otra parte, en el estudio de las empresas con frecuencia se utiliza el término eficiencia económica, en el que generalmente se asume que dos de sus componentes son la eficiencia técnica y la eficiencia asignativa. La forma de definirla se realiza a partir de suponer que una unidad económica produce lo máximo posible, dado un conjunto de insumos y una tecnología. La cuantificación de estas medidas se pude ver mediante tres perspectivas: 1) comparar por medio de unidades económicas similares, lo que indica la eficiencia relativa; 2) las medidas revelan variación entre las unidades económicas, en cuyo caso, el análisis se puede realizar para identificar los factores que causan las variaciones; y 3) el análisis tiene implicaciones de política económica para mejorar la eficiencia.

La medida de la eficiencia técnica tiene dificultades complejas, y la literatura provee un conjunto de metodologías ${ }^{1}$ tanto para el nivel microeconómico o empresas, como macroeconómico o a nivel industrial. A este respecto, en el estudio de la eficiencia un tema relevante es determinar el límite o frontera a partir de la cual se podrán comparar sus diferentes niveles. El concepto de frontera en la teoría microeconómica se determina por la función de producción y representa el máximo producto obtenible de acuerdo con un conjunto de insumos.

Así, actualmente el interés por tener una medida de eficiencia tiene dos razones: 1) porque se acepta que existen brechas entre suponer la eficiencia técnica teórica y la eficiencia técnica de la realidad empírica; y 2) existe una razón a priori, y es que hay una probabilidad alta de que, cuando existe ineficiencia técnica, esto influya en la eficiencia asignativa y tenga efecto negativo en la eficiencia económica.

En este contexto, el objetivo de este trabajo es obtener un indicador de niveles de eficiencia técnica que permita identificar la posición relativa de las entidades federativas de México respecto a la frontera eficiente. Asimismo, esto ofrece la posibilidad de responder a la interrogante de ¡cómo se está llevando a cabo la evolución de la eficiencia en las entidades

\footnotetext{
${ }^{1}$ Para medir la eficiencia se pueden utilizar dos técnicas: la de fronteras estocásticas (stochastic frontier analysis, SFA) y la de análisis de envolvente de datos (data envelopment analysis, DEA). La primera implica métodos econométricos y la segunda programación matemática.
} 
federativas, a partir de 1980? El estudio permite identificar las disparidades existentes entre las entidades federativas de México. El método que se emplea es el de fronteras estocásticas, el cual permite flexibilizar la estructura temporal de la ineficiencia técnica.

Esta investigación se divide de la siguiente forma: en el primer apartado se presentan los antecedentes teóricos sobre la medición de la eficiencia técnica; el segundo trata acerca de la metodología de fronteras estocásticas que se emplea, en particular el modelo de Battese y Coelli (1995); en el tercero se estima la eficiencia técnica para las entidades federativas mostrando la evolución temporal y las desigualdades; en el cuarto se presenta un conjunto de propuestas de política económica que pueden incidir en la mejora de la eficiencia por medio de la cantidad y calidad de los factores productivos. Por último se presentan las principales conclusiones obtenidas durante la investigación.

\section{Contexto de análisis de la eficiencia técnica}

El concepto básico para analizar la eficiencia se encuentra en la descripción de la tecnología de producción. Ésta se puede representar por una función de producción, una isocuanta, o una función de costo o beneficio. De ello, uno de los primeros estudios sobre el análisis de las fronteras de producción se encuentra en Farrell (1957), donde se define la eficiencia de la empresa, estableciendo que está constituida por dos componentes, la eficiencia técnica -que refleja su habilidad para obtener la máxima producción dado un conjunto de insumos- y la eficiencia en precios (o asignativa), la cual refleja su habilidad para utilizar los insumos en proporciones óptimas, de acuerdo con sus respectivos precios.

Asimismo, los enfoques para medir la eficiencia técnica difieren en función de la tecnología de producción, los supuestos y las técnicas de estimación que se proponen para cada modelo. Por ejemplo, Aigner y Chu (1968) consideraron la estimación de una función de producción de frontera paramétrica con una tecnología tipo Cobb-Douglas, usando una muestra de $\mathrm{N}$ empresas y definiendo un modelo con la siguiente forma funcional:

$$
\ln (Y)=x_{i} \beta-u_{i} ; i=1,2, \ldots N
$$

Donde $\ln (Y)$ es el logaritmo de la producción para la i-ésima empresa, $x_{i}$ es un vector renglón de tamaño $(\mathrm{k}+1)$, cuyo primer elemento es 1 , y el resto de los elementos están expresados en logaritmos de los $\mathrm{k}$ insumos usados por la firma i-ésima, $\beta=\left(\beta_{1}, \beta_{2} \ldots, \beta_{k}\right)$ es un vector columna de tamaño $(\mathrm{k}-1)$ de parámetros desconocidos a ser estimados y $u_{i}$ es una 
variable aleatoria no negativa, asociada con la ineficiencia técnica en la producción de las empresas de la industria involucrada.

El cociente de producción observado por la i-ésima empresa relativo a la producción, determinado por el vector de insumos, $x_{i}$, se utiliza para definir la eficiencia técnica de la i-ésima empresa:

$$
T E=\frac{y_{\mathrm{i}}}{\exp \left(x_{i} B\right)}=\frac{\exp \left(x_{i} B_{i}-u B_{i}\right)}{\exp \left(x_{i} B\right)}=\exp \left(-u_{i}\right)
$$

Esta medida es output orientada, y una medida de la eficiencia técnica la cual toma valores entre cero y uno, indicando la magnitud de la producción de la i-ésima empresa, relativa al output que puede producir una empresa completamente eficiente usando algún vector de insumos.

Posteriormente, Aigner et al. (1977) sugirieron una nueva aproximación a la estimación de fronteras de producción, en la idea de enlazar la teoría con el trabajo empírico. Su aproximación supone especificar un término de error con dos componentes, uno de tipo normal y otro con una distribución unilateral.

Así, Aigner et al. (1977) y Meeusen y Van Den Broeck (1977) propusieron de manera independiente la estimación de la frontera de producción estocástica, siguiendo un modelo teórico de la forma:

$$
Y i=f\left(X_{i}, \beta\right)+\varepsilon_{i} ; \quad \varepsilon_{i}=v_{i}-u_{i} ; \quad i=1,2, \ldots, N
$$

Donde $Y i$ es la cantidad de producto obtenido y $f\left(X_{i} \beta\right)$ la forma funcional correspondiente a la tecnología de producción, $X_{i}$ es un vector de insumos y $\beta$ representa los parámetros del modelo. $\varepsilon_{1}$ se refiere al término de error, compuesto de dos partes, la primera de ellas un componente simétrico $v_{i}$-distribuido como una normal con media cero y varianza $\sigma^{2}{ }_{\mathrm{v}}^{-}$, que permite variaciones aleatorias de la frontera de las empresas y captura el efecto de los errores de medida, otro ruido estadistico, y choques fuera de control de los agentes económicos. El componente $u_{i}$ captura los efectos de la ineficiencia relativa a la frontera estocástica. ${ }^{2}$ Los parámetros son estimados por máxima verosimilitud, dados los supuestos de la distribución de los términos de error.

Entre los primeros artículos empíricos en los que se propuso explicar los efectos de la ineficiencia se encuentran los de Pitt y Lee (1981) y Kalirajan (1981). Estos artículos proponen dos etapas: en la primera in-

${ }^{2}$ El análisis de fronteras estocásticas -al ser un método paramétrico, y frente al análisis envolvente de datos- permite obtener estimadores eficientes o scores eficientes de los productores individuales. Dado que los scores varían entre productores, pueden relacionar características como tamaño y localización. Así, es posible identificar las fuentes de ineficiencia. Finalmente, al análisis de fronteras estocásticas provee una poderosa herramienta para examinar efectos de intervención, como los que se dan después de aplicar una medida de política económica. 
volucran la especificación y estimación de la frontera estocástica de producción y la predicción de los efectos de la ineficiencia técnica, con el supuesto de que los efectos de esa ineficiencia están distribuidos de manera idéntica; la segunda etapa implica la especificación de un modelo para predecir los efectos de la ineficiencia técnica. Este método de estimación lo critican Battese y Coelli (1995), quienes argumentan que contradice el supuesto de que los efectos de la ineficiencia están distribuidos por igual en la frontera estocástica.

Trabajos como los de Kumbhakar et al. (1991) y Huang y Liu (1994) proponen modelos de efectos de ineficiencia técnica que involucran funciones de frontera estocástica. Los parámetros de la frontera estocástica y el modelo de ineficiencia técnica se estiman simultáneamente proporcionando el supuesto de distribución apropiado, con datos de corte transversal sobre una muestra de empresas. En años recientes, se identifica una importante cantidad de trabajos donde se aplica el cálculo de la eficiencia, entre otros: Battese y Coelli (1995), Gumbau y Maudos (1996, 2002), Álvarez (2001), Maudos et al. (2000), Pastor y Serrano (2000), Álvarez y Becerril (2005), Jayasuriya y Wodon (2007), Paxton (2007), Becerril et al. (2007), Braun y Cullmann (2008), Álvarez et al. (2008) y Becerril et al. (2009a). Todas estas aplicaciones se utilizan en diferentes contextos regionales y, la mayoría, mediante la aplicación del análisis de fronteras estocásticas. Así, a continuación se presenta el modelo propuesto por Battese y Coelli (1995), que se utiliza en esta investigación.

\section{Modelo de fronteras estocásticas}

Con frecuencia, el análisis de eficiencia se aplica en la investigación económica, tanto en el ámbito de la eficiencia técnica -mediante la función de producción-, o bien en el contexto de la eficiencia asignativa y económica, tomando como base la frontera de costos o la de beneficios. Así, la eficiencia productiva se alcanza cuando las economías maximizan la producción con los insumos disponibles. Su cálculo permite disponer de información sobre el comportamiento de la economía durante el periodo analizado y comparar los resultados de las economías objeto de estudio. Si las economías no están aprovechando de manera adecuada sus recursos, pueden realizar ajustes que les posibiliten incrementar su producción y mejorar su eficiencia.

En la aplicación empírica que se lleva a cabo en esta investigación, dado que se estima una función de producción, se analiza la eficiencia técnica siguiendo las medidas introducidas por Farrell (1957), que son de tipo radial. En este caso, los indicadores de ineficiencia se miden por medio de las desviaciones respecto de la frontera de producción, lo que 
permite aproximar a nivel empírico la función de producción. En este trabajo, en particular, se aproxima la frontera de producción mediante el modelo planteado por Battese y Coelli (1995), que flexibiliza la estructura del tipo de variación que sigue la eficiencia, frente a otros trabajos e incluso a la versión anterior de ellos mismos (1992).

En su modelo de 1995 se considera la función de producción estocástica para un panel de datos de tipo:

$$
\left.Y_{i t}=\exp \left(X_{i t}^{\prime} \beta\right)+v_{i t}-u_{i t}\right) ; \quad \mathrm{i}=1, \ldots, \mathrm{N} ; \quad \mathrm{t}=1, \ldots, \mathrm{T}
$$

Donde $Y_{i t}$ es la producción en el periodo $t$-ésimo y para la empresa $i$, $X_{i t}$ un vector (1xk) de variables explicativas asociadas con la $i$-ésima empresa en el $t$-ésimo periodo de observación y, $\beta$ un vector $(\mathrm{kx} 1)$ de parámetros desconocidos a ser estimados. En cuanto a los dos componentes que constituyen el término de error, $v_{i t}$ son los errores aleatorios independientes y distribuidos de manera idéntica como una normal, con media cero y varianza $\sigma_{v}^{2}, \mathrm{~N}\left(0, \sigma_{\mathrm{v}}^{2}\right)$, independientemente distribuidos de $u_{i p}$ los cuales son variables aleatorias no negativas, asociadas con la ineficiencia técnica de la producción.

Por su parte, $u_{i t}$ se compone por variables aleatorias no negativas, asociadas a la ineficiencia técnica en producción, se asume distribuido de forma independiente y se obtiene de una distribución normal truncada ${ }^{3}$ (en cero), con media $Z_{i t} \delta$ y varianza $\sigma^{2}$.

$Z_{i t}$ es un vector $(1 \mathrm{xm})$ de variables explicativas asociadas a la ineficiencia técnica a lo largo del tiempo, y $\delta$ es un vector $(m x 1)$ de coeficientes desconocidos de variables de ineficiencia de una empresa específica.

La ecuación (1) especifica la frontera de producción estocástica en términos de los valores de producción originales. A su vez, la ineficiencia técnica, $u_{i \nu}$, es función de un conjunto de variables explicativas, $Z_{i \nu}$ y un vector de coeficientes desconocidos, $\delta$. Así, la ineficiencia técnica se expresa como:

$$
u_{i t}=Z_{i t} \delta+W_{i t}
$$

Donde, $W_{i t}$ es una variable aleatoria que sigue una distribución normal truncada en $-Z_{i t} \delta$ con media cero y varianza $\sigma^{2}$. Las ecuaciones (1)-(2) se

${ }^{3}$ Battese y Coelli (1995) suponen que la ineficiencia técnica sigue una distribución normal truncada en cero. Puesto que la ineficiencia técnica sólo puede reducir la producción por debajo de la frontera, es necesario suponer distribuciones simétricas asociadas a dicho término, donde son igualmente aceptables las distribuciones half-normal y exponencial. Sin embargo, en diversos trabajos empíricos (por ejemplo Gumbau y Maudos, 1996) se demuestra que los resultados obtenidos, siguiendo cualquiera de las distribuciones mencionadas, son similares. 
estiman simultáneamente siguiendo el método de máxima verosimilitud, ${ }^{4}$ para obtener una eficiencia técnica de la forma:

$$
E T_{i t}=\frac{E\left(Y_{i t}^{*} / u_{i t} X_{i t}\right)}{E\left(Y_{i t}^{*} / u_{i t}=0, X_{i t}\right)}=\exp \left(-u_{i t}\right)
$$

Donde $Y^{*}$ es la producción, que es igual a $Y_{i t}$ cuando la variable dependiente no está transformada e igual a $\exp \left(Y_{i t}\right)$, cuando ésta se expresa en logaritmos. Por tanto, la eficiencia técnica se calcula como el cociente del nivel de producción obtenido respecto del máximo alcanzable, dadas las cantidades de los inputs (es decir, cuando $u_{i t}=0$ ). Asimismo, su valor oscila entre 0 y 1 , siendo éste último caso el más favorable.

Este modelo permite flexibilizar la estructura temporal de la ineficiencia técnica, frente a los trabajos anteriores que también siguen un patrón de variación temporal común para todas las empresas, entre los que se encuentra la versión anterior de Battese y Coelli (1992). Para ello se define la ecuación (2), que analiza los efectos que determinan la ineficiencia mediante una función explícita de factores específicos de cada empresa, entre los que se pueden encontrar las variables explicativas de la función de producción (ecuación 1), efectos fijos (individuales o temporales), así como cualquier variable susceptible de generar cambios en la ineficiencia técnica.

En el tratamiento de los problemas econométricos, como el anterior, el uso de un panel de datos disminuye los problemas de multicolinealidad y permite el tratamiento del problema de variables omitidas (Hsiao 1986). Asimismo, es importante destacar las propiedades que implica el método de máxima verosimilitud. Sin embargo, los problemas derivados de la posible endogeneidad de los regresores tienen difícil solución en el contexto de técnicas de frontera estocástica, cuando se asumen supuestos distribucionales específicos para el término de ineficiencia. ${ }^{5}$ En cuanto a la matriz de varianzas y covarianzas de los residuos, este modelo no tiene en cuenta la posible existencia de problemas de autocorrelación y heteroscedasticidad en alguna de las componentes del término de error. Finalmente, entre las ventajas que caracterizan el modelo de Battese y Coelli (1995) destacan las siguientes: 1) la variación temporal de la eficiencia técnica se puede someter a contraste; 2) es un modelo de componentes del error, pues este tipo de modelos emplea la información de que la ineficiencia es de una sola cola, en cuyo caso las empresas eficientes tienen mayor influencia en la estimación de la frontera; 3) la variación de la eficiencia técnica sigue un patrón diferenciado entre empresas; y 4)

\footnotetext{
${ }^{4}$ La función de verosimilitud y sus derivadas parciales con respecto a los parámetros del modelo se presentan en Battese y Coelli (1993), donde la primera se expresa en función de los parámetros de la varianza $\sigma_{s}^{2}=\sigma^{2}$ y $\sigma^{2} / \sigma_{s}^{2}$, siguiendo la parametrización en Battese y Corra (1977).

${ }^{5}$ Gumbau y Maudos (1996) hacen referencia a esta limitación.
} 
permite analizar la correlación entre los insumos y la eficiencia técnica (Álvarez, 2001: 74).

\section{Estimación de la eficiencia técnica en las entidades federativas}

El panel de datos considerado contiene información temporal del periodo 1980-2003 para las entidades federativas de México. La variable de producción, $Y_{i \uparrow}$ la representa el producto interno bruto (PIB) a precios de 1993 , los insumos de producción, los $x_{i p}$, se incorporan mediante la inversión, que corresponde a la formación bruta de capital fijo ( precios de 1993, y el empleo, L, que hace referencia al personal ocupado. Las fuentes estadísticas de las que se han obtenido las bases de datos proceden de los censos económicos del Instituto Nacional de Estadística y Geografía (INEGI). ${ }^{6}$

Siguiendo el modelo de Battese y Coelli (1995), que se desarrolló en el apartado anterior, se estima la eficiencia técnica en las entidades federativas de México en los años 1980, 1985, 1988, 1993, 1998 y 2003. La tecnología se representa mediante una función de producción translogarítmica de la forma:

$$
\begin{aligned}
& \text { In } Y_{i t}=\beta_{0}+\sum_{j=1}^{2} \beta_{j} \operatorname{In}\left(X_{j i t}\right)+\sum_{j=1}^{2} \sum_{h=1}^{2} \beta_{j h} \operatorname{In}\left(X_{j i t}\right) \operatorname{In}\left(X_{h i t}\right)+V_{i t}-U_{i t} \\
& \mathrm{i}=1, \ldots, 32 \text { entidades federativas } \\
& \mathrm{t}=1980, \ldots, 2003
\end{aligned}
$$

Donde $Y_{i t}$ es el producto y $X_{i t}$ es un vector que hace referencia a los inputs considerados (j,h ésimos empleo, L, y capital, K). $V_{i t}$ es el error aleatorio y $U_{i t}$ representa el término de ineficiencia. Éste, a su vez, se define mediante la ecuación:

$$
U_{i t}=\delta_{0}+\delta_{1} T+\delta_{2} T^{2}+\sum_{i=1}^{31} \lambda_{i} D_{i}+W_{i t}
$$

La ecuación de la ineficiencia incorpora una variable temporal (T), así como dummies individuales $\left(\mathrm{D}_{\mathrm{i}}\right)$, con el objetivo de controlar las diferencias no observadas entre las entidades federativas, dado que estos componentes también pueden influir en la eficiencia y, por último, el error aleatorio $W_{i t}$ La medida de la eficiencia técnica de Farrell (1957) se estima a partir de la expresión:

\footnotetext{
${ }^{6}$ En el Anexo 1 se exponen los estadísticos descriptivos correspondientes a las bases de datos empleadas en el presente trabajo, así como sus tasas de crecimiento.
} 


$$
E T_{i t}=\exp \left(-U_{i t}\right)=\exp \left[-\left(\delta_{0}+\delta_{1} T+\delta_{2} T^{2}+\sum_{i=1}^{31} \lambda_{i} D_{i}\right)-W_{i t}\right]
$$

De manera que la eficiencia técnica se calcula como el cociente del nivel de producción obtenido respecto del máximo alcanzable, dadas las cantidades de los insumos (es decir, cuando $U_{i t}$ ). Su valor oscilará entre 0 y 1 , siendo éste último caso el más eficiente.

En el cuadro 1 se presenta un conjunto de contrastes de razón de verosimilitud $(\lambda)$, que contribuyen a seleccionar la forma funcional más adecuada, tras decidir cuáles hipótesis nulas que se plantean serán aceptadas. En el primer contraste se rechaza la hipótesis nula de que se prefiere la forma funcional Cobb-Douglas a la translogarítmica. A continuación se contrasta la existencia de ineficiencia técnica en el término de error. Puesto que se rechaza la hipótesis de que el parámetro $\gamma$ es igual a cero, se confirma la necesidad de incorporar la ineficiencia técnica en la función de producción y el hecho de que una función de producción media supone una representación inadecuada de los datos. Por último, los contrastes tercero, cuarto y quinto consideran la hipótesis de que la ecuación de la ineficiencia no es función de los regresores considerados, y se confirma la significatividad de las variables que explican la ineficiencia técnica, incluidos los efectos individuales, ya que dichas hipótesis se rechazan.

\section{Cuadro 1}

\section{Contrastes de especificación}

\begin{tabular}{lcrrl}
\hline \multicolumn{1}{c}{ Hipótesis nula } & $\begin{array}{c}\text { Log. } F \\
\text { verosimilitud }\end{array}$ & Valor & Valor critico & \multicolumn{1}{c}{$\begin{array}{c}\text { Decisión } \\
-95 \%\end{array}$} \\
\hline $\mathrm{H}_{\mathrm{o}}=\beta_{\mathrm{KL}}=\beta_{\mathrm{L}^{2}}=\beta_{\mathrm{K}^{2}}=0$ & $-92,884$ & 60,53 & 7,815 & Rechazo \\
$\mathrm{H}_{\mathrm{o}}=\gamma=\delta_{0}=\ldots=\delta_{33}=0$ & $-143,188$ & 161,139 & 49,229 & Rechazo \\
$\mathrm{H}_{\mathrm{o}}: \delta_{1}=\delta_{2}=0$ & $-76,909$ & 28,581 & 5,991 & Rechazo \\
$\mathrm{H}_{0}=\delta_{3}=\ldots=\delta_{33}=0$ & $-94,624$ & 64,011 & 46,979 & Rechazo \\
$\mathrm{H}_{0}=\delta_{1}=\ldots=\delta_{33}=0$ & $-105,064$ & 84,89 & 46,979 & Rechazo \\
\hline
\end{tabular}

Fuente: Elaboración propia con base en la estimación de las ecuaciones 4 y 5 .

El estadístico $\lambda$ se calcula como: $\lambda=-2\left[\log \left(f\right.\right.$, verosimiliud $\left.\left(\mathrm{H}_{0}\right)\right)-\log \left(f\right.$, verosimiliud $\left.\left.\left(\mathrm{H}_{1}\right)\right)\right]$, que se distribuye según una chi-cuadrado con grados de libertad iguales al número de parámetros que se igualan a cero en la hipótesis nula. En el contraste cuya hipótesis nula considera $\gamma=0$ el estadístico $\lambda$ sigue una distribución chi-cuadrado mixta. Así, los valores críticos se obtienen de Kodde y Palm (1986), tabla 1: 1246.

Con base en el estudio de Battese y Coelli (1995), simultáneamente se lleva a cabo la estimación por máxima verosimilitud de las ecuaciones (4)-(5) mediante el uso del programa estadístico Frontier 4.1 (Coelli, 1996). Los resultados se presentan en el cuadro 2. 


\section{Cuadro 2}

Función de producción translogarítmica (Battese y Coelli, 1995)

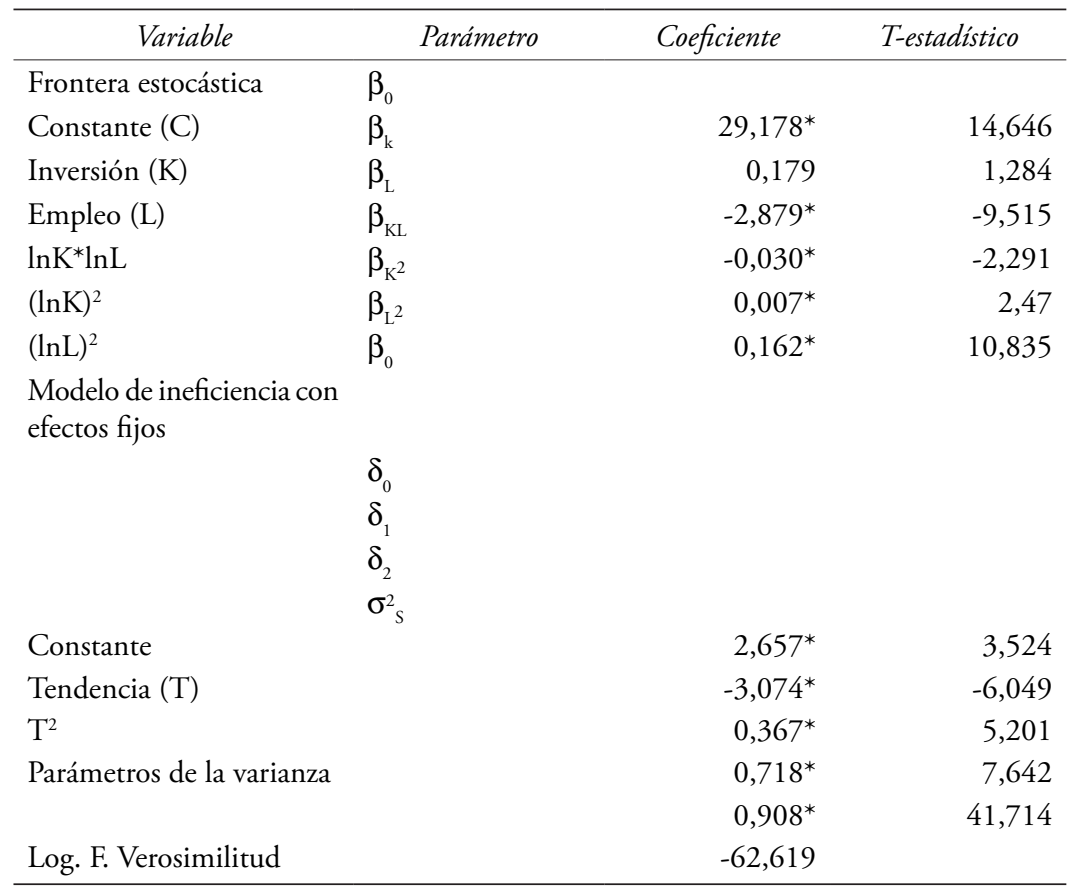

Fuente: Elaboración propia con base en la estimación de las ecuaciones 4 y 5.

*Parámetro significativo al $95 \%$.

Puesto que todas las hipótesis nulas se rechazan, se estima el modelo de frontera estocástica especificando la función de producción translogarítmica y la ecuación de ineficiencia propuestas. La varianza de los parámetros se expresa en términos de $\gamma=\frac{\sigma^{2}}{\sigma^{2}+\sigma^{2}}$ y $\sigma^{2}{ }_{S}=\sigma^{2}{ }_{v}+\sigma^{2}$, siendo $\sigma^{2}{ }_{v}$ y $\sigma^{2}$ las varianzas en las distribuciones de $V_{i t}^{v}$ y $U_{i t}$, respectivamente. Así, el valor del parámetro $\gamma$ indica que la proporción de la varianza de $U_{i t}$ sobre el error compuesto total es de $71.8 \%$, y muestra el error cometido al utilizar las funciones de producción medias en las que se ignoran las diferencias en eficiencia. Por último, los valores de la eficiencia técnica para las entidades federativas durante el periodo 1980-2003 obtenidos a partir del modelo de frontera estocástica, que se consigue mediante la implementación de la expresión (6), y con el uso del programa estadístico Frontier 4.1 (Coelli, 1996), se presentan en el Anexo estadístico 2. En el siguiente apartado se analiza su evolución temporal. 


\subsection{Evolución temporal de la eficiencia técnica}

En la gráfica i se presenta la evolución de la eficiencia técnica durante el periodo objeto de estudio (1980-2003). Los resultados obtenidos muestran la trayectoria que ha experimentado el agregado de la economía mexicana. Acerca de esto, no se debe olvidar que el 25 de julio de 1985 el peso se devaluó $20 \%$ respecto al nivel registrado el día anterior, y que el 5 de agosto entró en vigor un sistema de flotación regulada del tipo de cambio controlado, en sustitución del desliz uniforme en vigor desde diciembre de 1982 (Banco de México, 1985). Estos acontecimientos, entre otros, derivaron en situaciones desfavorables para la economía mexicana, cuyos efectos trascendieron en su comportamiento hasta 1988, año que estuvo determinado por la instrumentación del Pacto de Solidaridad Económica (PSE), aunado a una situación externa desfavorable, la cual se sumó a problemas internos de sequía y después por ciclones (Banco de México, 1988). Así, en este contexto, el indicador promedio de la eficiencia técnica de la economía mexicana paso de $0.55^{7}$ en 1980 a 0.82 en 1988 , y se mantuvo en niveles de alrededor de 0.8 durante el resto del periodo.

Cabe mencionar que entre los factores que afectaron adversamente las expectativas sobre la evolución de la economía del país en el futuro inmediato a 1993 y, por tanto, la demanda agregada y la producción, destaca la incertidumbre que prevaleció prácticamente a lo largo de todo el año, y que venía gestándose desde 1992, en torno a la ratificación del Tratado de Libre Comercio (TLC). La formación bruta de capital del conjunto de los sectores público y privado sufrió un retroceso en 1993, cuando se contrajo 1.4\% (Banco de México, 1993), lo que pudo influir en la posterior devaluación del peso.

El periodo termina con un nivel de eficiencia de 0.8 , lo que indica que es posible aumentar la producción aproximadamente en $20 \%$, con los factores productivos utilizados y la tecnología disponible. Cabe decir que este valor es superior a la eficiencia media del periodo, que fue de $77.78 \%$. La gráfica I muestra la evolución de la eficiencia técnica durante el periodo de análisis.

En el cuadro 3 se presenta el ranking por entidades federativas, atendiendo a los niveles de eficiencia técnica obtenidos al principio y final del periodo, así como la eficiencia media a lo largo del mismo. En este cuadro destaca que al principio la mitad de las entidades federativas se encontra-

${ }^{7}$ Este dato, correspondiente a 1980, se debe considerar con cautela, ya que es posible que no sólo se deba al comportamiento de la producción, la inversión y el empleo, sino que probablemente esté influido por problemas de captura de datos y de contabilidad, como documenta Esquivel (1999) respecto a la contabilidad que se llevó a cabo de la producción petrolera del golfo de México en las décadas de 1970 y 1980. Además, en esos años los vaivenes de los precios del petróleo -dice Esquivel- sin duda también afectaron la producción y el ingreso de los estados petroleros. 


\section{Gráfica I}

\section{Evolución de la eficiencia técnica media en México, 1980-2003}

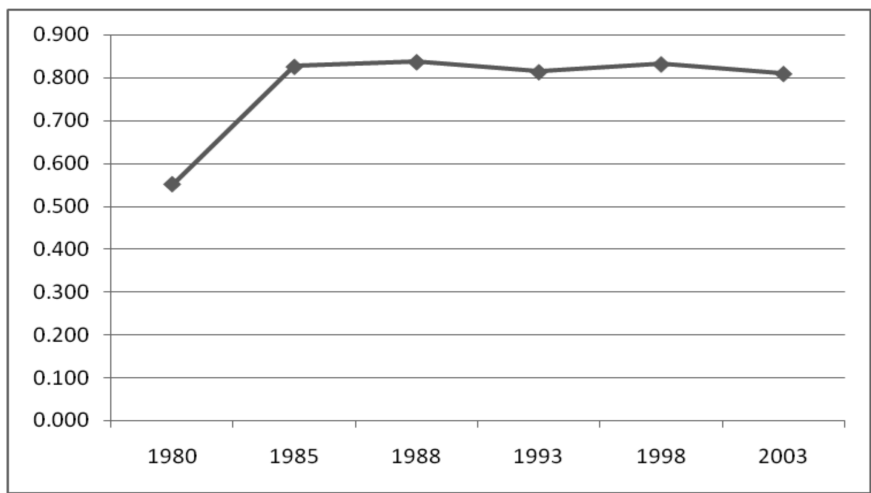

Fuente: Elaboración propia a partir de los datos de eficiencia técnica que aparecen en el Anexo estadístico 2.

ban con niveles de eficiencia menores al promedio (0.550); sin embargo, en el año 2003 esta situación mejoró: 10 estados que se encontraban por debajo de la media, en ese año aumentaron a 0.809. Esta información refleja una mejora relativa -respecto a la media- en la eficiencia técnica de las entidades federativas, lo cual se corrobora por la reducción de la varianza observada en 1980 y 2003. A lo largo del periodo la eficiencia promedio fue de $0.778,12$ entidades federativas se ubicaron por debajo de este valor, en orden descendente: Oaxaca, Campeche, Querétaro de Arteaga, Zacatecas, Coahuila de Zaragoza, Yucatán, Quintana Roo, Aguascalientes, Nayarit, Colima, Baja California Sur y Tlaxcala.

En la gráfica in se muestra el ranking de las entidades federativas en el año 2003, ordenadas de la menos a la más eficiente. Ésta refleja que los estados de Tlaxcala, Nayarit y Colima son los que muestran niveles de eficiencia técnica más baja, en tanto que en el otro extremo se encuentran Nuevo León, Distrito Federal y Veracruz.

La gráfica iII permite observar cómo ha evolucionado el indicador de cada entidad federativa en el año 2003 respecto a 1980, y muestra la diferencia entre su posición inicial y al final del periodo. Estados como Veracruz, Tabasco, México y el Distrito Federal presentan un incremento pequeño, sin embargo, muestran niveles altos de eficiencia. En el otro extremo, entidades como Tlaxcala, Quintana Roo, Coahuila y Campeche reflejan incrementos altos en su eficiencia técnica pero bajos niveles iniciales. 


\section{Cuadro 3}

\section{Ranking de eficiencia técnica de las entidades federativas, 1980-2003}

\begin{tabular}{|c|c|c|c|c|c|c|}
\hline Entidad federativa & $\begin{array}{c}\text { Eficiencia } \\
1980\end{array}$ & $\begin{array}{c}\text { Ranking } \\
1980\end{array}$ & $\begin{array}{c}\text { Eficiencia } \\
2003\end{array}$ & $\begin{array}{c}\text { Ranking } \\
2003\end{array}$ & $\begin{array}{l}\text { Eficiencia } \\
\text { media del } \\
\text { periodo }\end{array}$ & $\begin{array}{l}\text { Ranking } \\
\text { de } \\
\text { eficiencia } \\
\text { media del } \\
\text { periodo }\end{array}$ \\
\hline Aguascalientes & 0.26 & 26 & 0.780 & 25 & 0.617 & 28 \\
\hline Baja California & 0.704 & 15 & 0.884 & 6 & 0.863 & 12 \\
\hline Baja California Sur & 0.152 & 29 & 0.624 & 29 & 0.47 & 31 \\
\hline Campeche & 0.121 & 31 & 0.866 & 13 & 0.762 & 22 \\
\hline Chiapas & 0.818 & 7 & 0.875 & 9 & 0.888 & 6 \\
\hline Chihuahua & 0.78 & 9 & 0.894 & 5 & 0.876 & 9 \\
\hline $\begin{array}{l}\text { Coahuila de } \\
\text { Zaragoza }\end{array}$ & 0.001 & 32 & 0.762 & 26 & 0.673 & 25 \\
\hline Colima & 0.151 & 30 & 0.567 & 30 & 0.493 & 30 \\
\hline Distrito Federal & 0.888 & 2 & 0.904 & 2 & 0.911 & 4 \\
\hline Durango & 0.495 & 19 & 0.825 & 22 & 0.778 & 20 \\
\hline Guanajuato & 0.759 & 11 & 0.847 & 15 & 0.858 & 14 \\
\hline Guerrero & 0.619 & 16 & 0.843 & 16 & 0.829 & 16 \\
\hline Hidalgo & 0.55 & 17 & 0.803 & 23 & 0.804 & 17 \\
\hline Jalisco & 0.887 & 3 & 0.874 & 10 & 0.907 & 5 \\
\hline México & 0.906 & 1 & 0.896 & 4 & 0.921 & 1 \\
\hline Michoacán de & 0.784 & 8 & 0.833 & 18 & 0.861 & 13 \\
\hline \multicolumn{7}{|l|}{ Ocampo } \\
\hline Morelos & 0.444 & 21 & 0.832 & 20 & 0.78 & 19 \\
\hline Nayarit & 0.332 & 23 & 0.540 & 31 & 0.58 & 29 \\
\hline Nuevo León & 0.851 & 5 & 0.921 & 1 & 0.919 & 2 \\
\hline Oaxaca & 0.445 & 20 & 0.803 & 24 & 0.772 & 21 \\
\hline Puebla & 0.761 & 10 & 0.879 & 8 & 0.87 & 11 \\
\hline Querétaro de & 0.33 & 24 & 0.836 & 17 & 0.755 & 23 \\
\hline \multicolumn{7}{|l|}{ Arteaga } \\
\hline Quintana Roo & 0.197 & 27 & 0.829 & 21 & 0.644 & 27 \\
\hline San Luis Potosí & 0.516 & 18 & 0.832 & 19 & 0.798 & 18 \\
\hline Sinaloa & 0.756 & 12 & 0.854 & 14 & 0.871 & 10 \\
\hline Sonora & 0.749 & 13 & 0.883 & 7 & 0.877 & 8 \\
\hline Tabasco & 0.834 & 6 & 0.867 & 12 & 0.886 & 7 \\
\hline Tamaulipas & 0.737 & 14 & 0.869 & 11 & 0.857 & 15 \\
\hline Tlaxcala & 0.182 & 28 & 0.517 & 32 & 0.464 & 32 \\
\hline $\begin{array}{l}\text { Veracruz de Ignacio } \\
\text { de la Llave }\end{array}$ & 0.875 & 4 & 0.898 & 3 & 0.912 & 3 \\
\hline Yucatán & 0.327 & 25 & 0.709 & 28 & 0.663 & 26 \\
\hline Zacatecas & 0.402 & 22 & 0.748 & 27 & 0.732 & 24 \\
\hline Media aritmética & 0.55 & & 0.809 & & 0.778 & \\
\hline
\end{tabular}

Fuente: Elaboración propia a partir de los datos de eficiencia técnica que aparecen en cuadro 3 y en el Anexo estadístico 2.

Los números en las columnas de ranking indican la posición de la entidad federativa en orden descendente, donde el número 1 es el más eficiente. 


\section{Gráfica II}

\section{Eficiencia técnica de las entidades federativas, 2003}

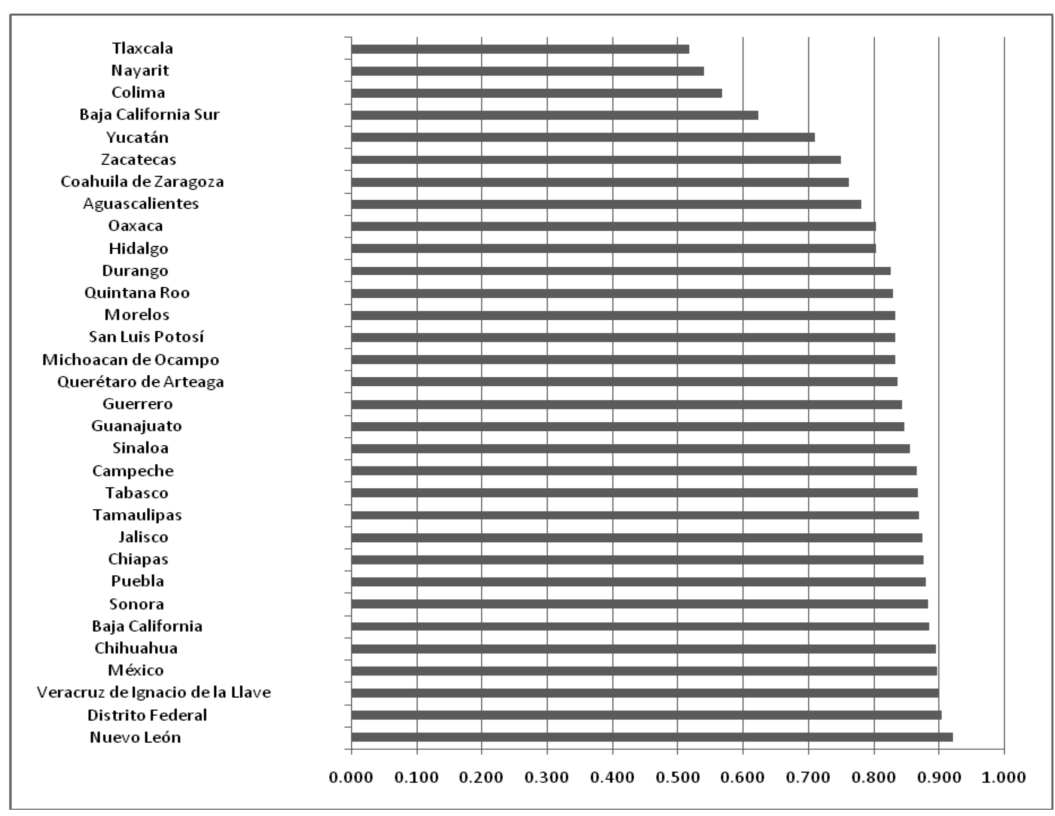

Fuente: Elaboración propia a partir de los datos de eficiencia técnica que aparecen en el Anexo estadístico 2.

Así, las gráficas II y III permiten observar las disparidades entre las entidades federativas al final del periodo y cómo han cambiado sus posiciones relativas en términos de eficiencia técnica, dejando ver las magnitudes del cambio.

La grafica IV muestra la convergencia en niveles de eficiencia de las entidades federativas durante el periodo 1980-2003, analizando la sigmaconvergencia a partir de la desviación típica del logaritmo del indicador de eficiencia, que permite extraer información sobre la dispersión existente a lo largo del tiempo. En la gráfica también aparece una evolución favorable, que indica que se han reducido las desigualdades en el periodo analizado, aunque este proceso no se ha producido de manera uniforme, dado que la gráfica muestra dos periodos bien diferenciados: el primero, que abarca los años 1980-1985, y el segundo a partir de este último año. Llama la atención la fuerte reducción que se observa de 1980 a 1985, año en que México entra al Acuerdo General sobre Aranceles Aduaneros y Comercio (GATT), y a partir de este último año, aunque todavía se observa una disminución, ésta es menor; lo cual significa que aunque se reducen las disparidades en el uso de los factores productivos, a partir de la 


\section{Gráfica III \\ Eficiencia técnica al inicio y final del periodo}

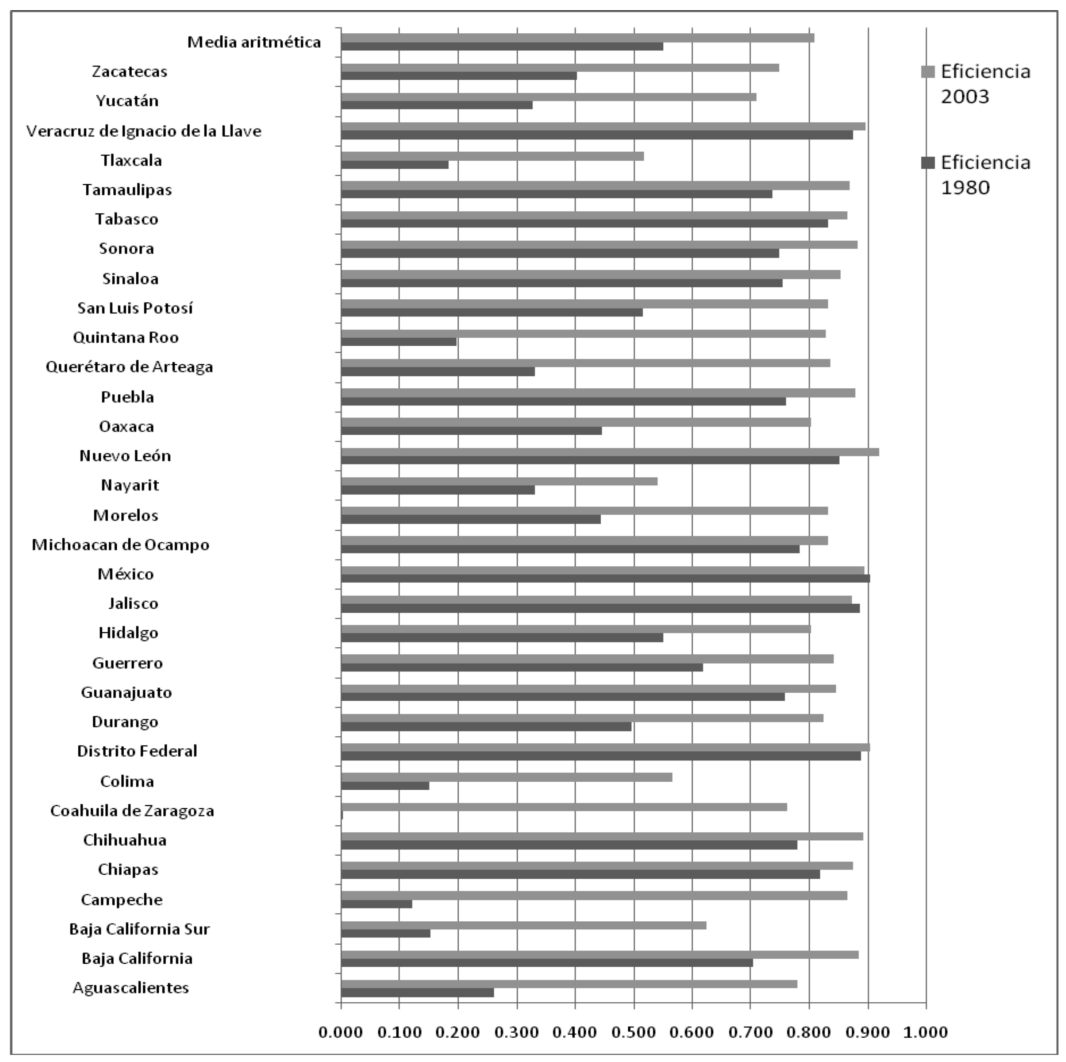

Fuente: Elaboración propia a partir de los datos de eficiencia técnica que aparecen en el Anexo estadístico 2.

apertura comercial de México, dicha reducción se hace más lenta. En la literatura para México sobre convergencia en renta, se identifican dos tipos de conclusiones: 1) en el modelo de industrialización orientado a las exportaciones se generó divergencia económica (Fuentes et al., 2003); y 2) dicho proceso ha reducido la velocidad de convergencia (Esquivel, 1999). De manera particular, Becerril et al. (2009b) encuentran que en el modelo de apertura comercial en México se ha dado un proceso de convergencia beta, es decir, aquellas entidades federativas que parten de menores niveles de eficiencia técnica experimentan mayores ganancias -en eficiencia-, sin embargo, éstas ha sido menores que en la última fase del modelo de industrialización vía sustitución de importaciones (periodo 1970-1993). 


\section{Gráfica IV}

\section{Sigma-convergencia de la eficiencia técnica en México, 1980-2003}

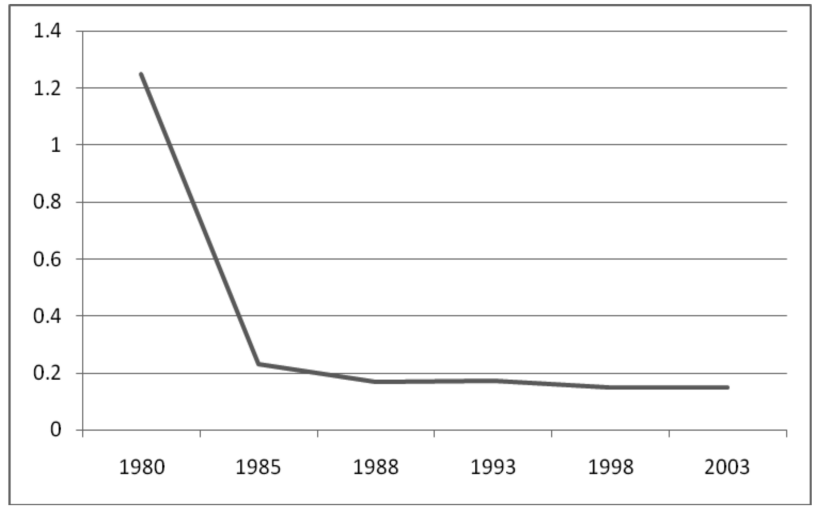

Fuente: Elaboración propia a partir de los datos de eficiencia técnica que aparecen en el Anexo estadístico 2.

A la luz de estos resultados, es posible elaborar un conjunto de propuestas de política económica que permitan incidir sobre los factores productivos, capital y empleo, ya que ellos son los inputs de la función de producción y, por tanto, la manera de combinarlos determina la eficiencia técnica. Así, una mayor calidad de éstos permitiría reducir la ineficiencia en la producción de las entidades federativas y del país. Con esta intención, en el siguiente apartado se propone un conjunto de acciones para incidir en la inversión y el empleo.

\section{Recomendaciones de política económica}

De acuerdo con los resultados obtenidos, se consideran algunas propuestas de política económica con el interés de incidir en acciones que pudieran hacer reflexionar a quienes formulan las políticas en México. De ello se desprende la importancia de avanzar en el diseño de estrategias que permitan mejorar el uso de los factores productivos: empleo y capital. De manera particular en lo que respecta a fuerza laboral, dado que es posible incrementar su productividad, ésta se debe incentivar a partir de incidir en el sistema educativo a partir del desarrollo y modernización de programas educativos, planes de estudio y métodos de enseñanza adecuados a los requerimientos del mercado laboral, a los cambios tecnológicos que impone la apertura comercial de México y su interacción con el mundo, y al mismo tiempo aumentar el gasto en educación, con el objetivo de elevar su competitividad. Los indicadores educativos de la fuerza laboral 
del país muestran que, en el año 2001, 61\% contaba con educación primaria, $17 \%$ con secundaria y $15 \%$ con educación terciaria. ${ }^{8}$ Estos datos reflejan una baja cualificación y calificación entre la población trabajadora del país. Cabe decir que en los últimos años el gasto en educación como porcentaje del PIB se ha incrementado, pasó de $5.2 \%$ en el año 2001 a 5.8\% en 2003, según datos del Banco Mundial (2007), y de acuerdo con datos de la Comisión Económica para América Latina y el Caribe (CEPAL, 2008), en 2005 fue de 5.6\%; sin embargo, el gasto en investigación y desarrollo (I\&D) como porcentaje del PIB es bajo, ya que pasó de $0.37 \%$ en el año 2000 a $0.40 \%$ en 2002 . Estos porcentajes son comparables en el ámbito latinoamericano con los que realiza la República Bolivariana de Venezuela (0.36\% y $0.39 \%$, respectivamente), pero por debajo de la República de Chile (ésta es la que tiene el mayor porcentaje de gasto en I\&D en América Latina) que ha invertido 0.53 y $0.70 \%$ de su PIB en esos años.

Respecto a la inversión, y de acuerdo con las recomendaciones de la Organización para la Cooperación y el Desarrollo Económico (ocDE, 2007), se sugiere dar mayor impulso a la inversión privada nacional y a la inversión extranjera directa mediante un sistema normativo y un mercado laboral flexibles y eficientes que permitan que estos capitales fluyan al sector productivo. Asimismo, para lograr un resultado favorable en la reducción de las disparidades en el uso de los factores productivos en las entidades federativas, se debe tener en cuenta un conjunto de acciones que incidan en algunos aspectos que se sugieren a continuación y que influyen en las decisiones de inversión. Desde la perspectiva del sector privado, y coincidiendo con el Banco Interamericano de Desarrollo (BID, 2004), se debe lograr una mayor estabilidad macroeconómica, ya que en términos de competitividad, México ocupa el lugar 48 en el ranking mundial. También se debe alcanzar la estabilidad política del país, así como el fortalecimiento ${ }^{9}$ credibilidad $^{10}$ institucional y normativa del sector público.

Asimismo, un conjunto de obstáculos a la participación privada provocan la ausencia o poca claridad en los servicios de regulación y coordi-

\footnotetext{
${ }^{8}$ De acuerdo con el World Economic Forum (2008), México se ubica en la posición 74 en el ranking de competitividad global en el rubro de competitividad en educación superior y adiestramiento.

${ }^{9}$ Desde la perspectiva de Rozas y Sánchez (2004), la existencia de instituciones débiles y una infraestructura pobre, pueden frenar el crecimiento económico a lo largo de una década, y mencionan que el grado de impacto de los gastos en infraestructura está positivamente relacionado con la calidad y el desarrollo de las instituciones.

${ }^{10}$ De acuerdo con Transparencia Internacional (2008), México ocupa el lugar 72 en el ranking global del índice de percepción de la corrupción y el lugar 14 en América, con una calificación de 3.6, lo que indica el grado de corrupción del sector publico, según la percepción de empresarios y analistas del país, entre 10 (percepción de ausencia de corrupción) y 0 (de muy corrupto).
} 
nación entre las entidades federativas. También desde el punto de vista económico y financiero, uno de los mayores problemas es la carencia de operadores privados capaces de ofrecer suficientes garantías técnicas y financieras para llevar a cabo los proyectos.

Finalmente, es importante no perder de vista la degradación y agotamiento de los recursos naturales en México, derivados de su uso continuo en los procesos productivos, con la finalidad de alcanzar un uso más eficiente de los factores productivos y un crecimiento ambientalmente sustentable y sostenible a largo plazo, acorde con los principios de la Agenda 21 y del Protocolo de Kioto. Su consideración radica en el hecho de que en el país, en el año 2002, y de acuerdo con el INEGi (2002), el efecto del daño ambiental representó $10.5 \%$ del piB; es decir, los costos en que se incurrió por su degradación y agotamiento, en tanto que únicamente $5.3 \%$ de estos costos representaron el gasto realizados para prevenir o disminuir el daño ambiental generado por las actividades productivas.

De esta manera, con las acciones conjuntas entre el gobierno, el sector privado y con la participación de la fuerza laboral, es posible aumentar la eficiencia técnica de las entidades federativas y reducir sus disparidades interestatales en México, con una perspectiva de crecimiento de largo plazo.

\section{Conclusiones}

En esta investigación se ha estimado la eficiencia técnica en las entidades federativas de México durante el periodo 1980-2003, siguiendo el modelo planteado por Battese y Coelli (1995). La evidencia obtenida muestra que la evolución que presenta ha sido estable durante el periodo objeto de estudio. A pesar de ello, existe la posibilidad de aumentar la producción en promedio aproximadamente $20 \%$ si se logra un uso más eficiente de los factores productivos. Los resultados obtenidos aportan información valiosa acerca del comportamiento en las distintas entidades federativas, dando un posicionamiento de cada una de ellas de acuerdo con sus niveles de eficiencia. De esta manera, al principio del periodo destacan el Estado de México, Jalisco y el Distrito Federal como los más eficientes, mientras que en el año 2003, Nuevo León, Distrito Federal, Veracruz y el Estado de México se mantienen en las primeras posiciones del ranking de eficiencia técnica.

La evolución temporal de la eficiencia técnica muestra que a partir de 1985 se mantiene un comportamiento relativamente estable, sin embargo, el estudio permite observar que los estados que parten de una posición menos eficiente tienen un crecimiento alto al final del periodo y, por el contrario, las entidades federativas que al inicio de periodo tenían un nivel 
alto de eficiencia presentan menores incrementos al final del periodo de estudio. Esto sugiere el cumplimiento de la hipótesis de convergencia planteada por Barro y Sala-i-Martín (1992) y Sala-i-Martín (1994, 1996a, 1996b) que expresa que si las economías son similares respecto a las preferencias y tecnología, entonces las economías pobres crecerán más rápido que las ricas. En el caso de convergencia en eficiencia, Becerril et al. (2007, 2009a) lo corroboran y esta investigación, mediante la sigma-convergencia, también confirma que hay una reducción en las disparidades en el uso de los factores productivos, siendo menos intensa en el modelo de industrialización orientado a las exportaciones implementado en México.

Así, el cálculo de la eficiencia técnica de las entidades federativas permite la posibilidad de reflexionar sobre las políticas públicas en México y sugerir algunas más. Las implicaciones se relacionan con el hecho de que aunque con el paso del tiempo se ha venido haciendo un mejor uso de los factores productivos capital y empleo, que se han reducido las disparidades interestatales en su uso y que aún es posible mejorar la producción haciendo un uso más eficiente de los inputs productivos, esto puede ser posible mediante el diseño de políticas encaminadas a hacer un mejor uso de los factores productivos y a través de la mejora en la calidad de los mismos.

\section{Anexo estadístico 1 Bases de datos}

\begin{tabular}{lccc}
\hline \multicolumn{1}{c}{ Entidad federativa } & Producción & Inversión & Empleo \\
\hline Aguascalientes & 18.724 & -53.357 & 24.621 \\
Baja California & 13.589 & -54.257 & 22.073 \\
Baja California Sur & 13.357 & -60.143 & 21.528 \\
Campeche & 22.936 & -72.039 & 28.217 \\
Coahuila de Zaragoza & 9.633 & -61.658 & 20.752 \\
Colima & 9.19 & -65.406 & 18.829 \\
Chiapas & 0.311 & -64.777 & 21.279 \\
Chihuahua & 14.115 & -56.647 & 22.817 \\
Distrito Federal & 5.718 & -59.805 & 8.623 \\
Durango & 7.562 & -59.36 & 19.21 \\
Guanajuato & 10.451 & -60.388 & 22.058 \\
Guerrero & 7.725 & -53.989 & 19.276 \\
Hidalgo & 4.805 & -61.113 & 17.107 \\
Jalisco & 6.559 & -59.595 & 18.013 \\
México & 5.009 & -64.105 & 15.348 \\
Michoacán de Ocampo & 5.888 & -57.231 & 20.406 \\
Morelos & 11.387 & -59.585 & 21.267 \\
\hline
\end{tabular}


continúa anexo estadístico 1 ...

\begin{tabular}{lccr}
\hline \multicolumn{1}{c}{ Entidad federativa } & Producción & Inversión & Empleo \\
\hline Nayarit & 1.94 & -52.582 & 17.41 \\
Nuevo León & 10.451 & -61.673 & 15.148 \\
Oaxaca & 8.865 & -66.289 & 22.318 \\
Puebla & 9.184 & -61.818 & 18.297 \\
Querétaro de Arteaga & 16.677 & -59.962 & 22.488 \\
Quintana Roo & 30.001 & -46.382 & 31.815 \\
San Luis Potosí & 9.744 & -60.178 & 19.275 \\
Sinaloa & 5.911 & -58.357 & 20.295 \\
Sonora & 8.087 & -61.609 & 19.4 \\
Tabasco & -9.166 & -76.224 & 19.039 \\
Tamaulipas & 8.65 & -60.644 & 20.18 \\
Tlaxcala & 10.04 & -60.798 & 19.535 \\
Veracruz de Ignacio de la Llave & 2.016 & -64.852 & 14.5 \\
Yucatán & 10.301 & -66.787 & 20.059 \\
Zacatecas & 6.382 & -53.7 & 19.214 \\
Media & 9.251 & -60.478 & 20.012 \\
\hline
\end{tabular}

Fuente: Elaboración propia con base a información de los censos económicos, INEGI.

\section{Estadísticos descriptivos}

\begin{tabular}{lrrrr}
\hline \multicolumn{1}{c}{ Variables } & \multicolumn{1}{c}{ Media } & \multicolumn{1}{c}{$\begin{array}{c}\text { Desviación } \\
\text { típica }\end{array}$} & Minimo & Máximo \\
\hline Producción & 35734809.27 & 48357985.51 & 3668021 & 337756747 \\
Inversión & 323388237.8 & 1223526418 & 5906.633 & $1.121 \mathrm{E}+10$ \\
Empleo & 327778 & 427260 & 22631 & 2842874 \\
\hline
\end{tabular}

Fuente: Elaboración propia con base a información de los censos económicos, INEGI. 


\section{Anexo estadístico 2}

Eficiencia técnica en las entidades federativas, 1980-2003

Función de producción translogarítmica. Modelo de frontera estocástica (Battese y Coelli, 1995)

\begin{tabular}{|c|c|c|c|c|c|c|}
\hline Entidad federativalaños & 1980 & 1985 & 1988 & 1993 & 1998 & 2003 \\
\hline Aguascalientes & 0.26 & 0.568 & 0.619 & 0.699 & 0.78 & 0.78 \\
\hline Baja California & 0.704 & 0.907 & 0.903 & 0.885 & 0.892 & 0.884 \\
\hline Baja California Sur & 0.152 & 0.399 & 0.507 & 0.548 & 0.591 & 0.624 \\
\hline Campeche & 0.121 & 0.926 & 0.938 & 0.86 & 0.859 & 0.866 \\
\hline Coahuila de Zaragoza & 0.001 & 0.801 & 0.817 & 0.849 & 0.81 & 0.762 \\
\hline Colima & 0.151 & 0.519 & 0.559 & 0.539 & 0.625 & 0.567 \\
\hline Chiapas & 0.818 & 0.937 & 0.921 & 0.882 & 0.896 & 0.875 \\
\hline Chihuahua & 0.78 & 0.903 & 0.883 & 0.899 & 0.898 & 0.894 \\
\hline Distrito Federal & 0.888 & 0.929 & 0.909 & 0.911 & 0.923 & 0.904 \\
\hline Durango & 0.495 & 0.862 & 0.848 & 0.794 & 0.847 & 0.825 \\
\hline Guanajuato & 0.759 & 0.909 & 0.901 & 0.872 & 0.859 & 0.847 \\
\hline Guerrero & 0.619 & 0.889 & 0.905 & 0.856 & 0.864 & 0.843 \\
\hline Hidalgo & 0.55 & 0.905 & 0.895 & 0.835 & 0.837 & 0.803 \\
\hline Jalisco & 0.887 & 0.927 & 0.934 & 0.913 & 0.905 & 0.874 \\
\hline México & 0.906 & 0.946 & 0.942 & 0.913 & 0.922 & 0.896 \\
\hline Michoacán de Ocampo & 0.784 & 0.901 & 0.907 & 0.863 & 0.879 & 0.833 \\
\hline Morelos & 0.444 & 0.861 & 0.852 & 0.837 & 0.857 & 0.832 \\
\hline Nayarit & 0.332 & 0.709 & 0.685 & 0.594 & 0.621 & 0.54 \\
\hline Nuevo León & 0.851 & 0.96 & 0.926 & 0.928 & 0.928 & 0.921 \\
\hline Oaxaca & 0.445 & 0.888 & 0.886 & 0.792 & 0.817 & 0.803 \\
\hline Puebla & 0.761 & 0.915 & 0.907 & 0.862 & 0.894 & 0.879 \\
\hline Querétaro de Arteaga & 0.33 & 0.825 & 0.831 & 0.829 & 0.876 & 0.836 \\
\hline Quintana Roo & 0.197 & 0.457 & 0.685 & 0.839 & 0.858 & 0.829 \\
\hline San Luis Potosí & 0.516 & 0.861 & 0.87 & 0.839 & 0.868 & 0.832 \\
\hline Sinaloa & 0.756 & 0.913 & 0.911 & 0.907 & 0.883 & 0.854 \\
\hline Sonora & 0.749 & 0.917 & 0.917 & 0.892 & 0.903 & 0.883 \\
\hline Tabasco & 0.834 & 0.947 & 0.931 & 0.863 & 0.875 & 0.867 \\
\hline Tamaulipas & 0.737 & 0.909 & 0.886 & 0.864 & 0.879 & 0.869 \\
\hline Tlaxcala & 0.182 & 0.56 & 0.567 & 0.467 & 0.491 & 0.517 \\
\hline $\begin{array}{l}\text { Veracruz de Ignacio de } \\
\text { la Llave }\end{array}$ & 0.875 & 0.941 & 0.935 & 0.908 & 0.916 & 0.898 \\
\hline Yucatán & 0.327 & 0.737 & 0.736 & 0.715 & 0.753 & 0.709 \\
\hline Zacatecas & 0.402 & 0.803 & 0.862 & 0.773 & 0.803 & 0.748 \\
\hline Media & 0.55 & 0.826 & 0.837 & 0.813 & 0.832 & 0.809 \\
\hline Desviación típica & 0.277 & 0.156 & 0.124 & 0.12 & 0.106 & 0.107 \\
\hline
\end{tabular}

Fuente: Elaboración propia con base en la estimación de las ecuaciones 4 y 5 . 


\section{Bibliografía}

Aigner, Dennis J. y S. F. Chu (1968), "On Estimating the Industry Production Function", American Economic Review, 58 (4), American Economic Association, Pittsburgh, pp. 826-839.

Aigner, Dennis J. C., A. Knox Lovell y Peter Schmidt (1977), “Formulation and estimation of stochastic frontier production function models", Journal of Economics, 6, Springer, Berlín, pp. 21-37.

Álvarez, Inmaculada y Osvaldo Becerril (2005), "Influencia del capital público y de la inversión en educación sobre la eficiencia técnica en las economías europeas y catch-up tecnológico, 1980-2001", Quivera, nueva época, 1, Universidad Autónoma del Estado de México, Toluca, pp. 134-169.

Álvarez, Inmaculada, Osvaldo Becerril, Laura del Moral y Reyna Vergara (2008), "Aplicación del data envelopment análisis a la delimitación de la frontera tecnológica en México (1970-2003)", Enlaces: Revista del CES Felipe II, 8-1, pp. 1-18, http://www.cesfelipesegundo.com/revista/articulos2008/Inmaculada\%20Alvarez--corregido.pdf (consulta: 27/07/2010).

Álvarez, Rafael (2001), "Modelos con eficiencia técnica variante en el tiempo", en A. Álvarez (coord.), La medición de la eficiencia y la productividad, Pirámide, Madrid.

Banco de México (1985), Informe Anual, Banco de México, México.

Banco de México (1988), Informe Anual, Banco de México, México.

Banco de México (1993), Informe Anual, Banco de México, México.

Banco Mundial (2007), World Development Indicators 2007, Banco Mundial, Washington.

BID (Banco Interamericano de Desarrollo) (2004), "Análisis del contexto de inversión en infraestructura en América Latina y el Caribe 1995-2003", Background paper, BID, Washington. 
Barro, Robert J. y Xavier Sala-i-Martín (1992), “Convergence”, Journal of Political Economy, 100 (2), University of Chicago Press, Chicago, pp. 223-251.

Battese, George y Greg S. Corra (1977), "Estimation of a production frontier model: with application to the pastoral zone of Eastern Australia”, Australian Journal of Agricultural Economics, 21, Australian Agricultural and Resourse Economics Society Asia Pacific School of Economics And Government, Camberra, pp. 169-179.

Battese, George E. y Tim Coelli (1992), "Frontier production functions, technical efficiency and panel data: with application to paddy farmers in India", Journal of Productivity Analysis, 3, Springer, Doetinchem, Países Bajos, pp. 153-169.

Battese, George E. y Tim Coelli (1993), "A stochastic frontier production function incorporating a model for technical inefficiency effects", Working Paper in Econometrics and Applied Statistics 69/93, University of New England, Armidale.

Battese, George E. y Tim Coelli (1995), "A model for technical inefficiency effects in a stochastic frontier production function for panel data", Empirical Economics, 20, Institute for Advanced Studies, Viena, pp. 325-332.

Becerril, Osvaldo, Inmaculada Álvarez y Reyna Vergara (2007), “Disparidades en eficiencia técnica y convergencia en eficiencia en México: un análisis de frontera”, Quivera, 9 (2), Universidad Autónoma del Estado de México, Toluca, pp. 131-154.

Becerril, Osvaldo, Inmaculada Álvarez y Laura del Moral (2009a), "Disparidades en eficiencia técnica e influencia de las infraestructuras sobre la convergencia en eficiencia en México", Investigaciones Regionales (en prensa).

Becerril, Osvaldo, Inmaculada Álvarez y Laura del Moral (2009b), "Do infrastructures influence the convergence of efficiency in México?", Journal of Policy Modeling, 32, Society for Policy Modeling, Rhode-St-Genese, pp. 120-137.

Braun, Frauke G. y Astrid Cullmann (2008), "Key Parameters and Efficiency of Mexican Manufacturing -Are There Still Differences 
between the North and the South: An Application of Nested and Stochastic Frontier Panel Data Models", Discussion Papers 816, German Institute for Economic Research, Berlín.

CEPAL (Comisión Económica para América Latina y el Caribe) (2008), Anuario Estadístico de América Latina y el Caribe, 2008, CePaL, Santiago.

Coelli, Tim (1996), "A guide to Frontier Version 4.1.: a computer program for stochastic frontier production and cost function estimation", CEPA Working Paper 96/07, Armidale.

Esquivel, Gerardo (1999), "Convergencia regional en México, 19401995”, El Trimestre Económico, 64 (4), 264, Fondo de Cultura Económica, México, pp. 725-761.

Farrell, Michael J. (1957), "The measurement of productive efficiency", Journal of the Royal Statistical Society, 120, Royal Statistical Society, Welwyn Garden City, pp. 253-290.

Fuentes Flores, Noe y Jorge Eduardo Arón y Mendoza Cota (2003), "Infraestructura pública y convergencia regional en México, 1980-1998”, Comercio Exterior, 53 (2), México, pp. 178-187.

Gumbau Albert, Mercedes y Joaquín Maudos (1996), "Eficiencia productiva sectorial en las regiones españolas: una aproximación frontera”, Revista Española de Economía, 13 (2), Asociación Española de Economía, Bilbao, pp. 239-260.

Gumbau Albert, Mercedes y Joaquín Maudos (2002), "The determinants of efficiency: the case of the Spanish industry", Applied Economics, 34, Routledge, Londres, pp. 1941-1948.

Hsiao, Cheng (1986), Analysis of Panel Data, Cambridge University Press, Cambridge.

Huang, Clief y Jin-Tan Liu (1994), "Estimation of a non-neutral stochastic frontier production function", Journal of Productivity Analysis, 5 (2), Springer, Doetinchem, Países Bajos, pp. 171-180.

INEGI (Instituto Nacional de Estadística, Geografía e Informática) (varios años), Censos económicos, INEGI, México. 
INEGI (Instituto Nacional de Estadística, Geografía e Informática) (2002), Sistema de cuentas económicas y ecológicas de México, SCEEM, INEGI, México.

Jayasuriya, Ruwan y Quentin Wodon (2007), "Efficiency in improving health and education outcomes: provincial and state-level estimates for Argentina and México (2007)", Estudios Económicos, 22 (1), El Colegio de México, México, pp. 57-97.

Kalirajan K. P. (1981), "An Econometric Analysis of Yield Variability in Paddy Production", Canadian Journal of Agricultural Economics, 29, Agricultural Economics Society, Victoria, pp. 283-294.

Kodde, David A. y Franz C. Palm (1986), "Wald Criteria for Jointly Testing Equality and Inequality Restrictions", Econometrica, 54 (5), Journal of the Econometric Society, Nueva York, pp. 12431248 .

Kumbhakar, Subal, C., J. Soumendra Ghosh y J. Thomas McGuckin (1991), "A Generalized Production Frontier Approach for Estimating Determinants of Inefficiency in U.S. Dairy Farms", Journal of Business \& Economic Statistics, 9 (3), American Statistical Association, Alejandría, pp. 279-286.

Maudos, Joaquín José Manuel Pastor y Lorenzo Serrano (2000), "Efficiency and productive specialization: An application to the Spanish regions", Regional Studies, 34 (9), Regional Studies Association. Seaford, pp. 829-842.

Meeusen, Wim y Julien van den Broeck (1977), "Efficiency estimation from Cobb-Douglas production with composed error", Internacional Economic Review, 18, University of Pennsylvania, Filadelfia, pp. 435-444.

OCDE (Organización para la Cooperación y el Desarrollo Económico) (2007), Getting it Right OECD Perspectives on Policy Challenges in Mexico, OCDE, Perspectives on Policy Challenges in Mexico, OCDE, París.

Pastor, José Manuel y Lorenzo Serrano (2000), “Crecimiento de la productividad y su descomposición en progreso técnico y cambio en eficiencia: una aplicación sectorial y regional en España (1964- 
93)”, Investigaciones Económicas, 35 (1), Fundación SEPI, Madrid, pp. 177-205.

Paxton, Julia (2007), "Technical Efficiency in a Semi-Formal Financial Sector: The Case of Mexico", Oxford Bulletin of Economics and Statistics, 69 (1), University of Oxford, Oxford, pp. 57-74.

Pitt, Mark M. y Lung-Fei Lee (1981), "The Measurement and Sources of Technical Inefficiency in the Indonesian Weaving", Industry Journal of Development Economics, 9 (1), Elsevier, Amsterdam, pp. 43-64.

Rozas, Patricio y Ricardo Sánchez (2004), Desarrollo de infraestructura y crecimiento económico: revisión conceptual, Naciones Unidas Santiago.

Sala-i-Martín, Xavier (1994), "La riqueza de las regiones. Evidencia y teorías sobre crecimiento regional y convergencia”, Moneda y Crédito, 198, Fundación Santander Central Hispano, вSCH, Madrid, pp. 13-80.

Sala-i-Martín, Xavier (1996a), "The classical aproach to convergence analysis", Economic Journal, 106, Royal Economic Society, St. Andrews, pp. 1019-1036.

Sala-i-Martín, Xavier (1996b), "Regional cohesion evidence and theories of regional growth and convergence", European Economic Review, 40, Elsevier, Amsterdam, pp. 1325-1352.

Schmidt, Peter (1976), "On the Statistical Estimation of Parametric Frontier Production Function", Review of Economics and Statistics, 58 (2), Harvard University's Kennedy School of Government, Massachusetts, pp. 238-239.

Transparencia Internacional (2008), Indice de percepción de la corrupción 2008, Transparencia Internacional, Berlín.

World Economic Forum (2008), Highligthts of the Global Competitiveness Report 2008-2009, World Economic Forum, Ginebra. 
Recibido: 26 de mayo de 2008.

Reenviado: 11 de mayo de 2009. Aceptado: 7 de diciembre de 2009.

Osvaldo Becerril-Torres. Es profesor del área de métodos cuantitativos. Investiga temas de convergencia y eficiencia. Actualmente se encuentra adscrito a la Facultad de Economía de la Universidad Autónoma del Estado de México. Entre sus últimas publicaciones destacan: en coautoría, "Disparidades en eficiencia técnica e influencia de las infraestructuras sobre la convergencia en eficiencia en México", Investigaciones Regionales (en prensa); "Disparidades en eficiencia técnica y convergencia en eficiencia en México: un análisis de frontera", Quivera, 2, Universidad Autónoma del Estado de México, México, pp. 131-154 (2007).

Inmaculada Álvarez-Ayuso. Es profesora del área de teoría económica, experta en temas de crecimiento económico, eficiencia, infraestructuras y econometría. Actualmente se encuentra adscrita a la Facultad de C. C. Económicas y Empresariales, Campus Cantoblanco, de la Universidad Autónoma de Madrid. Entre sus últimas publicaciones destacan: "Assessing the role of public policies in the analysis of EU convergence", International Journal of Economic Research (en prensa); "High capacity road networks and spatial spillovers: evidence of the Spanish case", Transportation Journal (en prensa).

Laura del Moral-Barrera. Es profesora del área de microeconomía y experta en temas de desarrollo regional agropecuario. Actualmente se encuentra adscrita a la Facultad de Economía de la Universidad Autónoma del Estado de México. Pertenece al cuerpo académico Economía de los sectores productivos e institucionales. Entre sus últimas publicaciones están: "El mercado de la carne de cerdo en canal en México", Análisis Económico, XXII (51), Universidad Autónoma Metropolitana, México, pp. 273-287; (2007); "Importaciones totales y de carne de cerdo en México en el contexto del TLCAN: un enfoque de corrección de error", Ciencia Ergo Sum, 14 (3), Universidad Autónoma del Estado de México, Toluca, pp. 263-271 (2007). 